\title{
Developing a Protocol for Medical Student-Organized Community-Based Hypertension Screening Programs
}

Vishal P. Varshney', Tyrone Harrison', Michal Szymczakowski', Matthew Grossi', Charlotte Jones².

\begin{abstract}
.
Background: Hypertension screening programs have been effective in raising awareness and identifying people who are otherwise unfamiliar with their disease. We aimed to develop a resource-minimal, evidence-based protocol for a novel medical student-organized hypertension-screening program capable of community implementation. Methods: Eighty-one medical students had their blood pressure measured once using an automated machine and once using the manual auscultatory method. Bland-Altman plots compared agreement between measurement techniques. Results: No significant difference between manual and automated techniques was noted in the measurement of systolic blood pressure, but was noted for diastolic blood pressure. Conclusions: In the context of a community-based screening program, automated and manual measurements may be used interchangeably to obtain an accurate measure of systolic blood pressure. A medical student-organized community-screening program is an effective way to screen large numbers of people in a short amount of time.
\end{abstract}

Keywords: Hypertension; Mass Screening; Blood Pressure Monitoring, Ambulatory; Students, Medical (Source: MeSH, NLM).

About the Author Vishal Varshney is a 3 rd year medical student at the University of Calgary, Calgary, $A B$, Canada.

\section{Introduction.}

The adverse effects of high blood pressure (hypertension) have been well established. ${ }^{1-3}$ Hypertension, defined in Canadian guidelines as an elevated blood pressure above $140 \mathrm{mmHg}$ systolic and/or $90 \mathrm{mmHg}$ diastolic, ${ }^{4}$ is estimated to cause more than one-eighth of all deaths worldwide, and is considered to be the leading risk factor for death in the world and a major risk factor for cerebrovascular, cardiac and other vascular diseases. ${ }^{3,5}$ While the level of hypertension control in Canada has risen considerably over the last 20 years, ${ }^{3}$ Thompson et al. revealed that at least $30 \%$ of Ontarians 18 years of age and older have uncontrolled hypertension or are unaware they have hypertension. ${ }^{2}$ Hypertension screening programs have been shown to be an effective way of raising awareness, identifying and managing patients who may otherwise not know of their disease..$^{6,7}$

Hypertension screening programs utilizing existing community resources to conduct door-to-door and mobile screening assessments have been in use since the 1970 's. ${ }^{8,9}$ More recently, community-based blood pressure programs, held in local pharmacies and specifically recruiting senior citizens, have shown that using a team-based approach with nurses, pharmacists and trained volunteers can effectively raise awareness, identify and manage seniors with hyper- tension.? Although not the focus of our study, these can be seen as a way to minimize white-coat hypertension, defined as a continuous elevation in blood pressure measurements in a clinical setting, which is estimated to have a prevalence of up to $25 \% .{ }^{10}$ Building on these successes, while also minimizing the resource-intense nature of these and other screening programs, our project aimed to develop an evidence-based protocol and technique for a novel medical student-organized hypertension screening program capable of being implemented in any public location.

Medical student-run public health initiatives have shown success in contributing to both medical education and patient care. "In our study, we used two methods to obtain blood pressures: manual auscultatory measurement (using calibrated aneroid machines and stethoscopes) and automated measurement (using a validated oscillometric blood pressure device), in order to determine if there were significant differences in blood pressure measurements between these techniques and to evaluate their ease of use in a community setting. The implications of our project are profound in that medical students are taught to take blood pressures early on in their training, and can conduct community-based blood pressure screening programs without needing significant economic resources. Adhering to a standard, evidence-based protocol means that any medi- 
cal school with basic equipment could coordinate screening programs in their communities, and be successful in reaching out to members of the community that may not have been identified as hypertensive otherwise.

\section{Methods.}

The protocol was approved by the Conjoint Health Research Ethics Board at the University of Calgary. Eight first year medical students met with their supervisor (CJ), reviewed CHEP guidelines and together designed and implemented the screening program. 81 2nd year medical students volunteered for the study through email. Consenting participants were excluded if they smoked, had caffeine or engaged in physical exercise one half-hour prior to measurement. Participants had their blood pressure measured twice, once with the automated technique ("Automated") using the BPTRU device (BPTRU Medical Devices, Coquitlam $B C$, Canada) and once with the manual auscultatory technique ("Manual") using calibrated aneroid machines (WelchAllyn Trimline Medical Products, Branchburg NJ, USA) and taken by medical students. The order in which their blood pressures were measured was randomly determined (www. random.org). Manual measurements were taken as per the Canadian Hypertension Education Program (CHEP) guidelines (4), and participants were required to rest a minimum of 5 minutes, before and between measurements with both devices. Three manual measurements were taken, with the last two measurements averaged for analysis. Six automated measurements were taken, with the last five measurements averaged for analysis.

Manual and automated measurements were compared and analyzed using Bland-Altman plots. In biostatistics, BlandAltman plots have been shown to analyze agreement between two different measurement types in a single person (12). As neither manual nor automated measurement techniques will give the "true" value of blood pressure, these plots help in determining whether the measurements are comparable. In the context of establishing a communitybased screening program, we sought to observe the agreement between automated measurements, which are quicker and easier to obtain, and manual measurements, which are commonly done in clinics. A post-event focus group was held with volunteers to determine how the process could be improved.

\section{Results.}

Eighty-one medical students consented to participate. Figure 1 shows all but four data points within the 95\% limits of agreement. The mean difference (manual - BpTRU in $\mathrm{mmHg}$ ) in systolic blood pressure (SBP) between devices was $-0.42 \mathrm{mmHg}$ ( $95 \%$ confidence intervals: -2.19 to 1.34 ).




There was no observable trend toward higher or lower readings regardless of the device used, average SBP or order of the blood pressure assessment. Variability was consistent across the range of SBP $(85-135 \mathrm{mmHg})$.

Figure 2 shows that all but two data points are within the $95 \%$ limits of agreement. The mean difference is significant (and clinically relevant), at $-4.92 \mathrm{mmHg}(95 \% \mathrm{Cl}:-6.45$ to -3.29). There was a tendency for the difference between methods to increase as diastolic blood pressure (DBP) increased. The variability around the mean difference remained constant across the range of DBP $(55-85 \mathrm{mmHg})$.

\section{Discussion.}

Bland and Altman demonstrated that, in the context of comparing blood pressure measurements, there could still be poor agreement between variables despite relatively high correlation coefficients. Correlation coefficients measure association and not agreement. ${ }^{12}$ This explains our reasoning for not presenting our data with regression analysis. BlandAltman plots are constructed with the average of values from the two measurements on the $x$-axis and the difference in values between the two measurements on the $y$ axis. Given that different hypertension guidelines highlight different management options for control of systolic and diastolic pressures, data was presented separately for diastolic and systolic pressures to observe the agreement between automated measurements, which are quicker to obtain, and manual measurements, which are commonly done in clinics - a main goal of our intended communitybased screening program.

The results indicate that there is no significant difference between manual and automated measurements in determining systolic pressure, but diastolic blood pressure measurements shows a larger, and significant, mean difference. The $95 \%$ confidence intervals also validate this. Increases in SBP have shown to be linearly related to cardiovascular mortality at all ages, and SBP has been recommended to become the major criterion for diagnosis, staging, and therapeutic management of hypertension. ${ }^{13,14}$ This suggests that in the context of a community-based screening program, manual or automated measurements can be used interchangeably and comparably for a subject to determine their systolic blood pressure, and thus, gain insight into their overall cardiovascular health.

Possible reasons as to why diastolic blood pressure measurements had a larger mean difference may be because Korotkoff sounds for diastolic pressure are difficult to auscultate in a community-based setting. ${ }^{15}$ In the manual auscultatory technique, five unique phases of sounds have been

Figure 2. Bland-Altman Plot for Diastolic Blood Pressure ( $n=81$ ). Mean difference $=-4.92 \mathrm{mmHg}$ (95\% $\mathrm{Cl}:-6.45$ to -3.29 ).

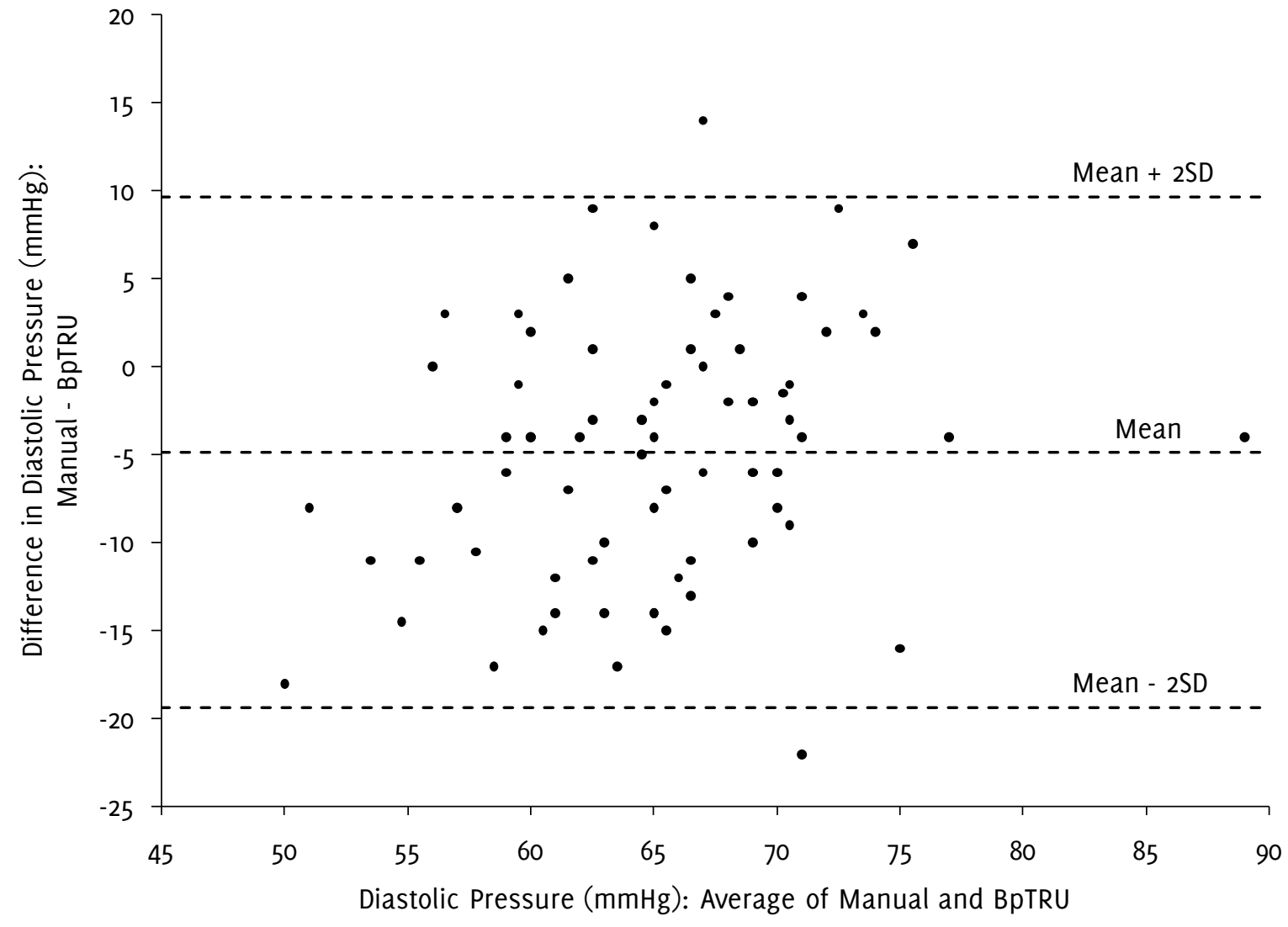


described: Phase I represents the first appearance of faint, repetitive, clear tapping sound which gradually increase in intensity for at least two consecutive beats (the systolic pressure); Phase II is a brief period where the sounds soften and "swish"; Phase III is the return of sharper sounds (clinical significance yet to be determined); Phase IV is the distinct abrupt muffling of sounds, which become soft and blowing in quality; and Phase $\mathrm{V}$ is the point at which all sounds finally disappear (the diastolic pressure). ${ }^{15}$ The "diastolic dilemma" is a well-described phenomenon that expresses uncertainty about the diastolic endpoint, in that Phase IV sounds may coincide with, or occur at pressures as much as $10 \mathrm{mmHg}$ higher than Phase $V$ sounds. ${ }^{15,16}$ Civen the subjective component in distinguishing between Phase IV and Phase $V$ sounds, especially in an environment with much ambient noise, manual diastolic pressures did show less agreement with automated diastolic pressures.

The possibility exists for a community-based hypertensionscreening program to possibly reduce the prevalence of white-coat hypertension, although further research is needed. Currently, ambulatory blood pressure monitoring is diagnostic for patients with elevated clinic blood pressures. ${ }^{17}$ If screening blood pressure in the community can remove the suggested conditioned response that results from endogenous pressor release incited in the clinical setting, 3,10 there could be a significant decrease in the number of false positive hypertension diagnoses that are solely based on clinic measurements. More research is suggested in this area.

We successfully accommodated 81 participants within a 2-hour span, with only 8 volunteers. Operational costs for our specific set-up were extremely minimal, with the only costs being those of the BPTRU machines. An area with much ambient noise and high traffic was specifically sought for our study so as to best simulate a community environment. The entire set-up and take-down process required minimal effort from volunteers, and was estimated to be easily reproducible in other settings, such as shopping malls, schools, community centers and office tower lobbies. The post-event focus group identified front-desk organization and further training with the automated machines as areas of improvement for future projects. The ease with which one can establish a medical student-organized hypertension screening program in the community was reassuring, and we look forward to further research comparing community-based blood pressure assessment with that of the standard office setting to further validate the protocol. Student-led programs such as this may prove to be of benefit the community and public health.

\section{References.}

1. Campbell NRC, Conradson HE, Kang J, Brant R, Anderson T. Automated assessment of blood pressure using BpTRU compared with assessments by a trained technician and a clinic nurse. Blood Press Monit 2005; 10(5):257.

2. Thompson A, Campbell NR, Cloutier L, Costello JA, Dawes M, Hickey J, et al. Tackling the burden of hypertension in Canada. Can Fam Physician 2008; $54(12): 1659-62$

3. Wilkins K, Campbell NRC, Joffres MR, McAlister FA, Nichol M, Quach S, et al. Blood pressure in Canadian adults. Health Rep 2010;21(1):37-46.

4. Rabi DM, Daskalopoulou SS, Padwal RS, Khan NA, Grover SA, Hackam DG, et al. The 2011 Canadian Hypertension Education Program recommendations for the management of hypertension: blood pressure measurement, diagnosis, assessment of risk, and therapy. Can J Cardiol 2011;27(4):415-33. e2.

5. Egan BM, Zhao Y, Axon RN. US trends in prevalence, awareness, treatment, and control of hypertension, 1988-2008. JAMA 2010;303(20):2043-50.

6. Joffres MR, Ghadirian P, Fodor JG, Petrasovits A, Chockalingam A, Hamet P. Awareness, treatment, and control of hypertension in Canada. Am J Hypertens 1997;10(10):1097-102.

7. Jones C, Simpson SH, Mitchell D, Haggarty S, Campbell N, Then K, et al. Enhancing hypertension awareness and management in the elderly: lessons learned from the Airdrie Community Hypertension Awareness and Management Program (A-CHAMP). Can J Cardiol 2008;24(7):561.

8. Tudor Hart J. Semicontinuous screening of a whole community for hypertension. Lancet 1970;296(7666):223-6.

9. Wilber JA, Barrow JG. Hypertension - a community problem. Am J Med 1972;52(5):653-63.

10. Botomino A, Martina B, Ruf D, Bruppacher R, Hersberger KE. White coat effect and white coat hypertension in community pharmacy practice. Blood Press Monit 2005;10(1):13-8.

11. Simpson SA, Long JA. Medical student-run health clinics: important contributors to patient care and medical education. J Gen Intern Med 2007;22(3):352-6.

12. Bland J, Altman DG. Statistical methods for assessing agreement between two methods of clinical measurement. Lancet 1986:327(8476):307-10.

13. Izzo Jr JL, Levy D, Black HR. Importance of systolic blood pressure in older Americans. Hypertension 2000;35(5):1021-4.

14. Pastor-Barriuso R, Banegas JR, DamiÃ jn J, Appel LJ, Guallar E. Systolic blood pressure, diastolic blood pressure, and pulse pressure: an evaluation of their joint effect on mortality. Ann Intern Med 2003;139(9):731-9.

15. Beevers G, Lip GYH, O'Brien E. Blood pressure measurement. BMJ 2001;322(7293):1043-7.

16. Short D. The diastolic dilemma. BMJ 1976;2(6037):685-6.

17. Polonia II, Gama CM, Silva JA, Amaral C, Martins LR, Bertoquini SE. Sequential follow-up clinic and ambulatory blood pressure evaluation in a low risk population of white-coat hypertensive patients and in normotensives. Blood Press Monit 2005;10(2):57-64.

\section{Acknowledgements}

To Leanne Ross, Research Coordinator and Statistician; help with statistical analysis and project coordination. To Reena Hansen, Kimchi Nguyen, Shawna Jensen, Nicholas Field, Laura Coughlan and Elizabeth Kelly, Third year medical students.

\section{Funding}

Calgary Medical Students Association (CMSA) Endowment Fund.

\section{Conflict of Interest Statement}

The Authors have no conflict of interest to disclose.

Cite as:

Varshney VP, Harrison T, Szymczakowski M, Grossi M, Charlotte J. Developing a Protocol for Medical Student-Organized Community-Based Hypertension Screening Programs. Int J Med Students 2013;1(1):8-11. 